\title{
The adolescence of cancer immunotherapy: from a difficult childhood to a pillar of modern anticancer therapy
}

\author{
Thorsten Fuereder (D)
}

Received: 4 May 2018 / Accepted: 9 May 2018 / Published online: 24 May 2018

(c) Springer-Verlag GmbH Austria, part of Springer Nature 2018

Cancer immunotherapy was born in the 1890s and raised by its father William Coley, who provoked an immune response by injecting a mixture of different bacteria into patients suffering from a variety of sarcomas. Although impressive responses were observed, the efficacy of Coley's toxin was unpredictable and lethal side effects occurred. Therefore, immunotherapy was shelved for several decades and alternative concepts such as radiation or chemotherapy were preferred. With the advent of molecular oncology, immunotherapy was rediscovered and enthusiastically pursued. Tumor-specific monoclonal antibodies were invented and Bacillus Calmette-Guérin (BCG) was introduced for the therapy of early bladder cancer. However, immunotherapy was still not easy: cytokine therapy with interleukin 2 for example resulted in responses in chemotherapy resistant melanoma, but these responses were hardly durable and associated with substantial toxicity. While skepticism rose within the scientific community in the 1980s, immunotherapy reached maturity in the late 1990s and has turned into a powerful tool for anticancer therapy. In the year 2013 cancer immunotherapy was selected as the scientific breakthrough of the year by the prestigious journal Science. Currently, more than 2000 immuno-oncology agents are in preclinical and more than 900 agents are in clinical development [1]. The landscape of immunotherapy comprises T-cell targeted modulators such as checkpoint inhibitors, cancer vaccines, oncolytic viruses, adoptive T-cell therapy such as chimeric antigen receptor (CAR) T-cell therapy among many others. Immunotherapy

\section{T. Fuereder, MD ( $₫)$}

Department of Internal Medicine I \& Comprehensive Cancer Center, Division of Clinical Oncology, Medical University of Vienna, Währinger Gürtel 18-20, 1090 Vienna, Austria thorsten.fuereder@meduniwien.ac.at became standard of care and the preferred treatment option in a variety of malignancies: in metastatic melanoma, which is regarded as almost unresponsive to dacarbazine chemotherapy, the Checkmate 066 and 067 trials set a new gold standard for this disease with a 3-year overall survival (OS) rate of $58 \%$ in the ipilimumab/nivolumab combination arm [2]. Likewise, in metastatic non-small cell lung cancer (NSCLC) long-term survival can be achieved by immunotherapy. Very recently, the Keynote 189 trial in stage IV nonsquamous NSCLC was presented [3]: It was demonstrated that the new paradigm is not chemotherapy or immunotherapy but both in combination. Chemotherapy plus pembrolizumab results in a 1-year OS rate of nearly $70 \%$ [3]. But not only patients suffering from solid tumors benefit from immunotherapy. Adoptive T-cell therapy with socalled CAR $T$ cells revolutionized the treatment of patients with a malignant hematologic disease as well. The Eliana trial demonstrated durable responses in patients suffering from refractory or relapsed acute lymphoblastic leukemia (ALL) [4].

However, immunotherapy cannot deny its roots and major challenges remain. We still lack a predictive biomarker and it is hardly possible to identify in advance which patients will benefit from immunotherapy. Additionally, novel response patterns such as pseudoprogression were observed and traditional imaging assessment rules are no longer valid for immunotherapy. Furthermore, immune-related adverse events require multidisciplinary management and both health care staff and patient education to prevent severe and potentially lethal side effects. Finally, with the success and broad access of cancer immunotherapy, economic challenges for the public health care systems have arisen.

Taken together, immunotherapy has revolutionized medical oncology and is now the fourth pillar of anti- 
cancer treatment, but it still has to transit from adolescence to adulthood in order to unleash its full potential.

Conflict of interest T. Fuereder declares that he has no competing interests.

\section{References}

1. Tang J, Shalabi A, Hubbard-Lucey VM. Comprehensive analysis of the clinical immuno-oncology landscape. Ann Oncol. 2018;29:84-91.

2. Larkin J, Chiarion-Sileni V, Gonzalez R, et al. Combined Nivolumab and Ipilimumab or monotherapy in untreated melanoma. NEngl J Med. 2015;373:23-34.
3. Gandhi L, Rodriguez-Abreu D, Gadgeel S, et al. Pembrolizumab plus chemotherapy in metastatic non-smallcell lung cancer. N Engl J Med. 2018; https://doi.org/10. 1056/nejmoal801005.

4. Maude SL, Laetsch TW, Buechner J, et al. Tisagenlecleucel in children and young adults with B-cell lymphoblastic leukemia. NEngl J Med. 2018;378:439-48.

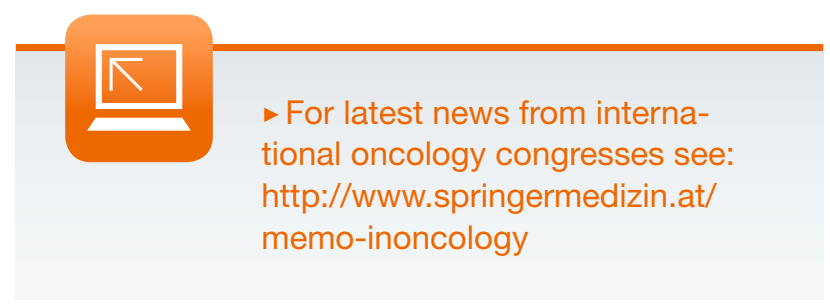

